\title{
First Results from the CERN Axion Solar Telescope
}

K. Zioutas, ${ }^{8}$ S. Andriamonje, ${ }^{2}$ V. Arsov,${ }^{13,4}$ S. Aune, ${ }^{2}$ D. Autiero, ${ }^{1, *}$ F. T. Avignone, ${ }^{3}$ K. Barth, ${ }^{1}$ A. Belov, ${ }^{11}$ B. Beltrán, ${ }^{6}$ H. Bräuninger, ${ }^{5}$ J. M. Carmona, ${ }^{6}$ S. Cebrián, ${ }^{6}$ E. Chesi, ${ }^{1}$ J. I. Collar, ${ }^{7}$ R. Creswick, ${ }^{3}$ T. Dafni, ${ }^{4}$ M. Davenport, ${ }^{1}$ L. Di Lella,${ }^{1, \dagger}$ C. Eleftheriadis, ${ }^{8}$ J. Englhauser, ${ }^{5}$ G. Fanourakis, ${ }^{9}$ H. Farach, ${ }^{3}$ E. Ferrer, ${ }^{2}$ H. Fischer, ${ }^{10}$ J. Franz, ${ }^{10}$ P. Friedrich, ${ }^{5}$ T. Geralis, ${ }^{9}$ I. Giomataris, ${ }^{2}$ S. Gninenko, ${ }^{11}$ N. Goloubev,${ }^{11}$ M. D. Hasinoff, ${ }^{12}$ F. H. Heinsius, ${ }^{10}$ D. H. H. Hoffmann, ${ }^{4}$ I. G. Irastorza, ${ }^{2}$ J. Jacoby,${ }^{13}$ D. Kang,${ }^{10}$ K. Königsmann, ${ }^{10}$ R. Kotthaus,${ }^{14}$ M. Krčmar, ${ }^{15}$ K. Kousouris, ${ }^{9}$ M. Kuster, ${ }^{5}$ B. Lakić, ${ }^{15}$ C. Lasseur, ${ }^{1}$ A. Liolios,${ }^{8}$ A. Ljubičić, ${ }^{15}$ G. Lutz, ${ }^{14}$ G. Luzón, ${ }^{6}$ D. W. Miller, ${ }^{7}$ A. Morales, ${ }^{6, \sharp}$ J. Morales, ${ }^{6}$ M. Mutterer, ${ }^{4}$ A. Nikolaidis, ${ }^{8}$ A. Ortiz, ${ }^{6}$ T. Papaevangelou, ${ }^{1}$ A. Placci, ${ }^{1}$ G. Raffelt, ${ }^{14}$ J. Ruz, ${ }^{6}$ H. Riege, ${ }^{4}$ M. L. Sarsa, ${ }^{6}$ I. Savvidis, ${ }^{8}$ W. Serber,${ }^{14}$ P. Serpico, ${ }^{14}$ Y. Semertzidis, ${ }^{4,8}$ L. Stewart, ${ }^{1}$ J. D. Vieira, ${ }^{7}$ J. Villar, ${ }^{6}$ L. Walckiers, ${ }^{1}$ and K. Zachariadou ${ }^{9}$

(CAST Collaboration)

\author{
${ }^{1}$ European Organization for Nuclear Research (CERN), Genève, Switzerland \\ ${ }^{2}$ DAPNIA, Centre d'Études Nucléaires de Saclay (CEA-Saclay), Gif-sur-Yvette, France \\ ${ }^{3}$ Department of Physics and Astronomy, University of South Carolina, Columbia, South Carolina, USA \\ ${ }^{4}$ GSI-Darmstadt and Institut für Kernphysik, Technische Universität Darmstadt, Darmstadt, Germany \\ ${ }^{5}$ Max-Planck-Institut für Extraterrestrische Physik, Garching, Germany \\ ${ }^{6}$ Instituto de Física Nuclear y Altas Energías, Universidad de Zaragoza, Zaragoza, Spain \\ ${ }^{7}$ Enrico Fermi Institute and KICP, University of Chicago, Chicago, Illinois, USA \\ ${ }^{8}$ Aristotle University of Thessaloniki, Thessaloniki, Greece \\ ${ }^{9}$ National Center for Scientific Research "Demokritos," Athens, Greece \\ ${ }^{10}$ Albert-Ludwigs-Universität Freiburg, Freiburg, Germany \\ ${ }^{11}$ Institute for Nuclear Research (INR), Russian Academy of Sciences, Moscow, Russia \\ ${ }^{12}$ Department of Physics and Astronomy, University of British Columbia, Vancouver, Canada \\ ${ }^{13}$ Johann Wolfgang Goethe-Universität, Institut für Angewandte Physik, Frankfurt am Main, Germany \\ ${ }^{14}$ Max-Planck-Institut für Physik (Werner-Heisenberg-Institut), Munich, Germany \\ ${ }^{15}$ Rudjer Bošković Institute, Zagreb, Croatia \\ (Received 31 October 2004; published 1 April 2005)
}

\begin{abstract}
Hypothetical axionlike particles with a two-photon interaction would be produced in the sun by the Primakoff process. In a laboratory magnetic field ("axion helioscope"), they would be transformed into xrays with energies of a few keV. Using a decommissioned Large Hadron Collider test magnet, the CERN Axion Solar Telescope ran for about 6 months during 2003. The first results from the analysis of these data are presented here. No signal above background was observed, implying an upper limit to the axionphoton coupling $g_{a \gamma}<1.16 \times 10^{-10} \mathrm{GeV}^{-1}$ at $95 \%$ C.L. for $m_{a} \lesssim 0.02 \mathrm{eV}$. This limit, assumption-free, is comparable to the limit from stellar energy-loss arguments and considerably more restrictive than any previous experiment over a broad range of axion masses.
\end{abstract}

DOI: 10.1103/PhysRevLett.94.121301

PACS numbers: 95.35.+d, 14.80.Mz, 96.60.Vg

Introduction. -Neutral pions, gravitons, hypothetical axions, or other particles with a two-photon interaction can transform into photons in external electric or magnetic fields, an effect first discussed by Primakoff in the early days of pion physics [1]. Therefore, stars could produce these particles by transforming thermal photons in the fluctuating electromagnetic fields of the stellar plasma $[2,3]$. In the laboratory or astrophysical $B$ fields, transitions between these particles and photons occur [4,5], an effect that can be observed in the laboratory [6], affect the propagation of cosmic $\gamma$ rays [7,8], and can modify the apparent brightness of distant astronomical sources [911]. Gravitons interact too weakly to be observable in these situations, while pions are too heavy. However, these ef- fects can be crucial for new particles, notably the pseudoscalar axions that arise in the context of the Peccei-Quinn solution to the strong $C P$ problem and are viable cold dark matter candidates $[6,12]$. Galactic axions are currently being sought by two large-scale Primakoff-type microwave cavity experiments [12]. Anomalous stellar energy loss by axion emission is constrained by the observed properties of globular cluster stars, implying $g_{a \gamma} \leqslant 10^{-10} \mathrm{GeV}^{-1}$ [3] for the axion-photon coupling, where the axion-photon interaction is written in the usual form $\mathcal{L}_{a \gamma}=$ $-\frac{1}{4} g_{a \gamma} F_{\mu \nu} \tilde{F}^{\mu \nu} a=g_{a \gamma} \mathbf{E} \cdot \mathbf{B} a$. Axions would also contribute to the magnetically induced vacuum birefringence, interfering with the corresponding QED effect $[5,13]$. The experiment PVLAS [14] apparently observes such an ef- 
fect far in excess of the QED expectation, although an interpretation in terms of axionlike particles requires a coupling strength far larger than existing limits.

On the other hand, the sun would be a strong axion source and thus offers a unique opportunity to actually detect these particles by taking advantage of their backconversion into x-rays in laboratory magnetic fields [4]. The expected solar axion flux at the earth due to the Primakoff process is $\Phi_{a}=g_{10}^{2} 3.67 \times 10^{11} \mathrm{~cm}^{-2} \mathrm{~s}^{-1}$ (where $g_{10} \equiv g_{a \gamma} 10^{10} \mathrm{GeV}$ ) with an approximate spectrum $\quad d \Phi_{a} / d E_{a}=g_{10}^{2} 3.821 \times 10^{10} \mathrm{~cm}^{-2} \mathrm{~s}^{-1} \times$ $\mathrm{keV}^{-1}\left(E_{a} / \mathrm{keV}\right)^{3} /\left(e^{E_{a} / 1.103 \mathrm{keV}}-1\right)$ and an average energy of $4.2 \mathrm{keV}$ [15]. Axion interactions other than the twophoton vertex would provide for additional production channels, but in the most interesting scenarios these channels are severely constrained, leaving the Primakoff effect as the dominant one [3]. In any case, it is conservative to use the Primakoff effect alone when deriving limits on $g_{a \gamma}$.

The conversion probability in a $B$ field in vacuum is [4] $P_{a \rightarrow \gamma}=\left(g_{a \gamma} B / q\right)^{2} \sin ^{2}(q L / 2)$, where $L$ is the path length and $q=m_{a}^{2} / 2 E_{a}$ is the axion-photon momentum difference. For $q L \lesssim 1$, where the axion-photon oscillation length far exceeds $L$, we have $P_{a \rightarrow \gamma}=\left(g_{a \gamma} B L / 2\right)^{2}$, implying an x-ray flux of

$$
\Phi_{\gamma}=0.51 \mathrm{~cm}^{-2} \mathrm{~d}^{-1} g_{10}^{4}\left(\frac{L}{9.26 \mathrm{~m}}\right)^{2}\left(\frac{B}{9.0 \mathrm{~T}}\right)^{2} .
$$

For $q L \gtrsim 1$ this rate is reduced due to the axionphoton momentum mismatch. The presence of a gas would provide a refractive photon mass $m_{\gamma}$, so that $q=\left|m_{\gamma}^{2}-m_{a}^{2}\right| / 2 E_{a}$. For $m_{a} \approx m_{\gamma}$ the maximum rate can thus be restored [16].

The first implementation of the axion helioscope concept was performed in [19]. More recently, the Tokyo axion helioscope [20] of $L=2.3 \mathrm{~m}$ and $B=3.9 \mathrm{~T}$ has provided the limit $g_{10}<6.0$ at $95 \%$ C.L. for $m_{a} \leqslant 0.03 \mathrm{eV}$ (vacuum) and $g_{10}<6.8-10.9$ for $m_{a} \lesssim 0.3 \mathrm{eV}$ (using a variable-pressure buffer gas) [21]. Limits from crystal detectors [22-24] are much less restrictive.

CAST experiment. - In order to detect solar axions or to improve the existing limits on $g_{a \gamma}$, an axion helioscope has been built at CERN by refurbishing a decommissioned Large Hadron Collider test magnet [25] which produces a magnetic field of $B=9.0 \mathrm{~T}$ in the interior of two parallel pipes of length $L=9.26 \mathrm{~m}$ and a cross-sectional area $A=$ $2 \times 14.5 \mathrm{~cm}^{2}$. The aperture of each of the bores fully covers the potentially axion-emitting solar core $(\sim 1 / 10$ th of the solar radius). The magnet is mounted on a platform with $\pm 8^{\circ}$ vertical movement, allowing for observation of the sun for $1.5 \mathrm{~h}$ at both sunrise and sunset. The horizontal range of $\pm 40^{\circ}$ encompasses nearly the full azimuthal movement of the sun throughout the year. The time the sun is not reachable is devoted to background measurements. A full cryogenic station is used to cool the superconducting magnet down to $1.8 \mathrm{~K}$ [26]. The hardware and the software of the tracking system have been precisely calibrated, by means of geometric survey measurements, in order to orient the magnet to any given celestial coordinates. The overall CERN Axion Solar Telescope (CAST) pointing precision is better than $0.01^{\circ}$ [27]. At both ends of the magnet, three different detectors have searched for excess $\mathrm{x}$-rays from axion conversion in the magnet when it was pointing to the sun. Covering both bores of one of the magnet's ends, a conventional time projection chamber (TPC) is looking for x-rays from "sunset" axions. At the other end, facing "sunrise" axions, a second smaller gaseous chamber with novel MICROMEGAS (micromesh gaseous structure-MM) [28] readout is placed behind one of the magnet bores, while in the other one a focusing $\mathrm{x}$-ray mirror telescope is working with a charge coupled device (CCD) as the focal plane detector. Both the CCD and the $\mathrm{x}$-ray telescope are prototypes developed for $\mathrm{x}$-ray astronomy [29]. The $\mathrm{x}$-ray mirror telescope can produce an "axion image" of the sun by focusing the photons from axion conversion to a $\sim 6 \mathrm{~mm}^{2}$ spot on the CCD. The enhanced signal-to-background ratio substantially improves the sensitivity of the experiment. A detailed account of the technical aspects of the experiment will be given elsewhere.

Data analysis and first results. - CAST operated for about 6 months, from May to November in 2003, during most of which time at least one detector was taking data. The results presented here were obtained after the analysis of the data sets listed in Table I. An independent analysis was performed for each data set. Finally, the results from all data sets are combined.

An important feature of the CAST data treatment is that the detector backgrounds are measured with $\sim 10$ times longer exposure during the nonalignment periods. The use

TABLE I. Data sets included in our result.

\begin{tabular}{lcccccc}
\hline \hline Data set & $\begin{array}{c}\text { Tracking exposure } \\
(\mathrm{h})\end{array}$ & $\begin{array}{c}\text { Background exposure } \\
(\mathrm{h})\end{array}$ & $\begin{array}{c}\left(g_{a \gamma}^{4}\right)_{\text {best fit }}( \pm 1 \sigma \text { error }) \\
\left(10^{-40} \mathrm{GeV}^{-4}\right)\end{array}$ & $\chi_{\text {null }}^{2} /$ d.o.f. & $\chi_{\text {min }}^{2} /$ d.o.f. & $\begin{array}{c}g_{a \gamma}(95 \% \text { C.L. }) \\
\left(10^{-10} \mathrm{GeV}^{-1}\right)\end{array}$ \\
\hline TPC & 62.7 & 719.9 & $-1.1 \pm 3.3$ & $18.2 / 18$ & $18.1 / 17$ & 1.55 \\
MM set A & 43.8 & 431.4 & $-1.4 \pm 4.5$ & $12.5 / 14$ & $12.4 / 13$ & 1.67 \\
MM set B & 11.5 & 121.0 & $2.5 \pm 8.8$ & $6.2 / 14$ & $6.1 / 13$ & 2.09 \\
MM set C & 21.8 & 251.0 & $-9.4 \pm 6.5$ & $12.8 / 14$ & $10.7 / 13$ & 1.67 \\
CCD & 121.3 & 1233.5 & $0.4 \pm 1.0$ & $28.6 / 20$ & $28.5 / 19$ & 1.23 \\
\hline \hline
\end{tabular}


of these data to estimate and subtract the true experimental background during sun tracking data is the most sensitive step in the CAST analysis. To assure the absence of systematic effects, the main strategy of CAST is the use of three independent detectors with complementary approaches. In the event of a positive signal, it should appear consistently, if strong enough, in each of the three detectors when it is pointing at the sun. In addition, an exhaustive recording of experimental parameters was done, and a search for possible background dependencies on these parameters was performed. A dependence of the TPC background on the magnet position was found, caused by its relatively large spatial movements at the far end of the magnet, which resulted in appreciably different environmental radioactivity levels. Within statistics, no such effect was observed for the sunrise detectors which undergo a much more restricted movement. To correct for this systematic effect in the TPC data analysis, an effective background spectrum is constructed only from the background data taken in magnet positions where sun tracking has been performed, and this is weighted accordingly with the relative exposure of the tracking data. Further checks have been performed in order to exclude any possible systematic effect. They were based on rebinning the data, varying the fitting window, splitting the data into subsets, and verifying the null hypothesis test in energy windows or areas of the detectors where no signal is expected. In general, the systematic uncertainties are estimated to have an effect of less than $\sim 10 \%$ in the final upper limits obtained.

For a fixed $m_{a}$, the theoretically expected spectrum of axion-induced photons has been calculated and multiplied by the detector efficiency curves (determined both by calculation and measurements). These spectra, which are proportional to $g_{a \gamma}^{4}$, are directly used as fit functions to the experimental subtracted spectra (tracking minus background) for the TPC and the MM. For these data, the fitting is performed by standard $\chi^{2}$ minimization. Regarding the CCD data, the analysis is restricted to the small area on the CCD where the axion signal is expected after the focusing of the x-ray telescope. During the data taking period of 2003, a continuous monitoring of the pointing stability of the x-ray telescope was not yet possible, therefore a signal area larger than the size of the sun spot had to be considered. Taking into account all uncertainties of the telescope alignment, the size of the area containing the signal was conservatively estimated to be $34 \times 71$ pixels $\left(54.3 \mathrm{~mm}^{2}\right)$. As in the other detectors, the background is defined by the data taken from the same area during the nontracking periods, but, alternatively, the background in the signal area was also determined by extrapolating the background measured during tracking periods in the part of the CCD not containing the sun spot. Both methods of background selection led to the same final upper limit on the coupling constant $g_{a \gamma}$. The resulting low counting statistics in the
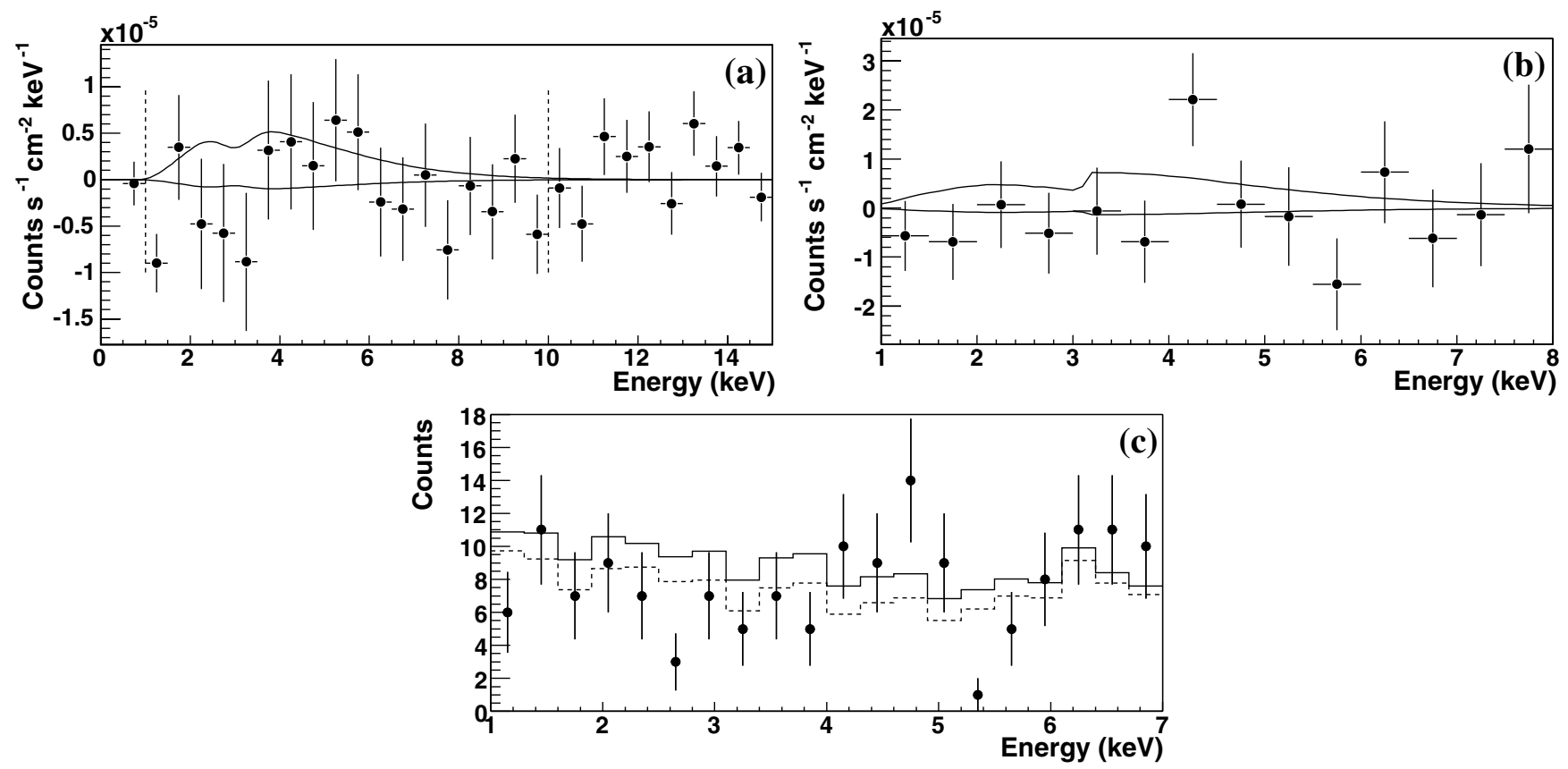

FIG. 1. Panels (a) and (b) show, respectively, the experimental subtracted spectrum of the TPC data set and MM data set A, together with the expectation for the best fit $g_{a \gamma}$ (lower curve) and for the 95\% C.L. limit on $g_{a \gamma}$. For (a) the vertical dashed lines indicate the fitting window. The structure at $3 \mathrm{keV}$ in the expected spectrum reflects the change in the efficiency curves due to the Ar $K$ edge of the detector gas mixtures. Panel (c) shows both the tracking (dots) and background (dashed line) spectra of the CCD data set, together with the expectation (background plus signal) for $g_{a \gamma}$ at its 95\% C.L. limit, in units of total counts in the restricted CCD area $\left(54.3 \mathrm{~mm}^{2}\right)$ in the tracking exposure time $(121.3 \mathrm{~h})$. 


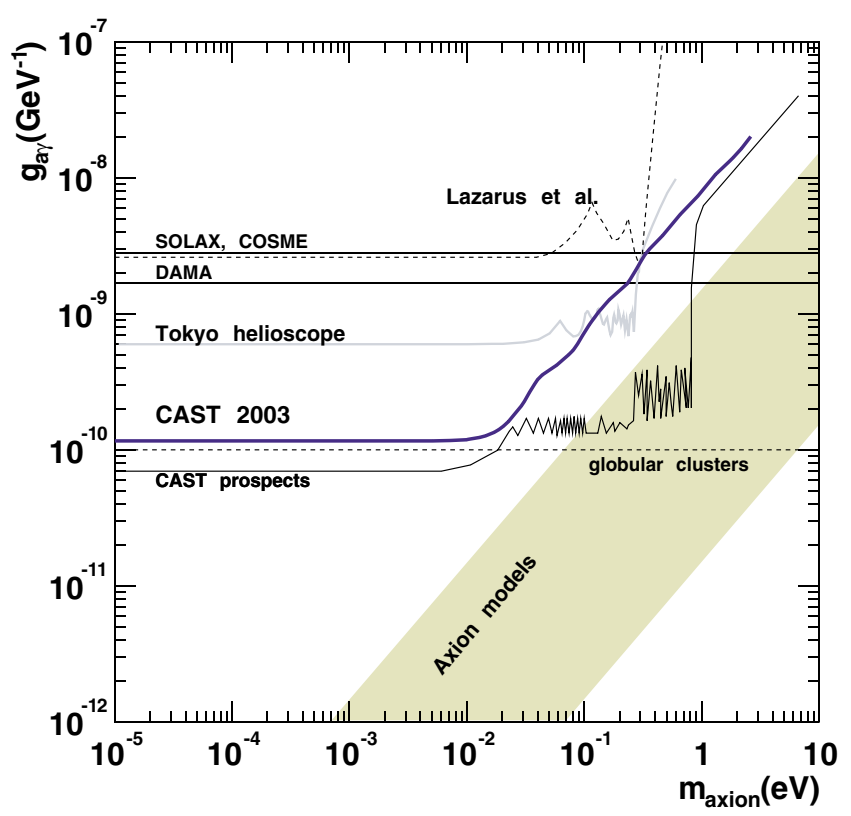

FIG. 2 (color online). Exclusion limit (95\% C.L.) from the CAST 2003 data compared with other constraints discussed in the introduction. The shaded band represents typical theoretical models. Also shown is the future CAST sensitivity as foreseen in the experiment proposal.

CCD required the use of a likelihood function in the minimization procedure, rather than a $\chi^{2}$ analysis.

The best fit values of $g_{a \gamma}^{4}$ obtained for each of the data sets are shown in Table I, together with their $1 \sigma$ error and the corresponding $\chi_{\min }^{2}$ values and degrees of freedom. Figure 1 shows the plots of some of those fits. Each of the data sets is individually compatible with the absence of any signal, as can be seen from the $\chi_{\text {null }}^{2}$ values shown in Table I. The excluded value of $g_{a \gamma}^{4}$ was conservatively calculated by taking the limit encompassing $95 \%$ of the physically allowed part (i.e., positive signals) of the Bayesian probability distribution with a flat prior in $g_{a \gamma}^{4}$. The described procedures were done using $g_{a \gamma}^{4}$ instead of $g_{a \gamma}$ as the minimization and integration parameter because the signal strength (i.e., number of counts) is proportional to $g_{a \gamma}^{4}$. The $95 \%$ C.L. limits on $g_{a \gamma}$ for each of the data sets are shown in the last column of Table I. They can be statistically combined by multiplying the Bayesian probability functions and repeating the previous process to find the combined result for the 2003 CAST data:

$$
g_{a \gamma}<1.16 \times 10^{-10} \mathrm{GeV}^{-1}(95 \% \text { C.L. }) .
$$

Thus far, our analysis was limited to the mass range $m_{a} \lesssim$ $0.02 \mathrm{eV}$ where the expected signal is mass-independent because the axion-photon oscillation length far exceeds the length of the magnet. For higher $m_{a}$, the overall signal strength diminishes rapidly and the spectral shape differs. Our procedure was repeated for different values of $m_{a}$ to obtain the entire 95\% C.L. exclusion line shown in Fig. 2.
Summary. - Our limit improves the best previous laboratory constraints [20] on $g_{a \gamma}$ by a factor 5 in our coherence region $m_{a} \lesssim 0.02 \mathrm{eV}$. This result excludes an important part of the parameter space not excluded by solar age considerations [30] and is comparable, in this range of masses, to the limit derived from stellar energyloss arguments. A higher sensitivity is expected from the 2004 data with improved conditions in all detectors, which should allow us to surpass the astrophysical limit. In addition, starting in 2005, CAST plans to take data with a varying-pressure buffer gas in the magnet pipes, in order to restore coherence for axion masses above $0.02 \mathrm{eV}$. The extended sensitivity to higher axion masses will allow us to enter into the region shown in Fig. 2 which is especially motivated by axion models [31].

We thank CERN for hosting the experiment and for the contributions of J. P. Bojon, F. Cataneo, R. Campagnolo, G. Cipolla, F. Chiusano, M. Delattre, F. Formenti, M. Genet, J. N. Joux, A. Lippitsch, L. Musa, R. De Oliveira, A. Onnela, C. Rosset, and B. Vullierme. We thank, in particular, F. James for his advice concerning the statistical treatment of the data. We acknowledge support from NSERC (Canada), MSES (Croatia), CEA (France), BMBF (Germany), GSRT (Greece), RFFR (Russia), CICyT (Spain), NSF (USA), and the European Union project ILIAS.

*Present address: Institut de Physique Nucléaire, Lyon, France.

${ }^{\dagger}$ Present address: Scuola Normale Superiore, Pisa, Italy.

† Deceased.

${ }^{\S}$ Permanent address: Brookhaven National Laboratory, NY, USA.

[1] H. Primakoff, Phys. Rev. 81, 899 (1951).

[2] D. A. Dicus et al., Phys. Rev. D 18, 1829 (1978).

[3] G. G. Raffelt, Annu. Rev. Nucl. Part. Sci. 49, 163 (1999).

[4] P. Sikivie, Phys. Rev. Lett. 51, 1415 (1983); 52, 695(E) (1984).

[5] G. Raffelt and L. Stodolsky, Phys. Rev. D 37, 1237 (1988).

[6] S. Eidelman et al., Phys. Lett. B 592, 1 (2004).

[7] D. S. Gorbunov, G. G. Raffelt, and D. V. Semikoz, Phys. Rev. D 64, 096005 (2001).

[8] C. Csaki et al., J. Cosmol. Astropart. Phys. 05 (2003) 005.

[9] C. Csaki, N. Kaloper, and J. Terning, Phys. Rev. Lett. 88, 161302 (2002).

[10] E. Mörtsell and A. Goobar, J. Cosmol. Astropart. Phys. 04 (2003) 003.

[11] B. A. Bassett, Astrophys. J. 607, 661 (2004).

[12] R. Bradley et al., Rev. Mod. Phys. 75, 777 (2003).

[13] L. Maiani, R. Petronzio, and E. Zavattini, Phys. Lett. B 175, 359 (1986).

[14] G. Cantatore et al., in Proceedings of the 5th International Workshop on the Identification of Dark Matter, Edinburgh, UK, 2004 (to be published). 
[15] The spectrum in [16] has been changed to that proposed in [17], however, with a modified normalization constant to match the total axion flux used here, which is predicted by a more recent solar model [18].

[16] K. van Bibber et al., Phys. Rev. D 39, 2089 (1989).

[17] R. J. Creswick et al., Phys. Lett. B 427, 235 (1998).

[18] J. N. Bahcall, M. H. Pinsonneault, and S. Basu, Astrophys. J. 555, 990 (2001).

[19] D. M. Lazarus et al., Phys. Rev. Lett. 69, 2333 (1992).

[20] S. Moriyama et al., Phys. Lett. B 434, 147 (1998).

[21] Y. Inoue et al., Phys. Lett. B 536, 18 (2002).

[22] F. T. Avignone et al., Phys. Rev. Lett. 81, 5068 (1998).

[23] A. Morales et al., Astropart. Phys. 16, 325 (2002).

[24] R. Bernabei et al., Phys. Lett. B 515, 6 (2001).

[25] K. Zioutas et al., Nucl. Instrum. Methods Phys. Res., Sect. A 425, 480 (1999).
[26] K. Barth et al., Proceedings of the 2003 Cryogenic Engineering Conference (CEC) and Cryogenic Materials Conference (ICMC) [Adv. Cryog. Eng. 49B, 168 (2004)].

[27] http://cast.web.cern.ch/CAST/edited_tracking.mov

[28] Y. Giomataris et al., Nucl. Instrum. Methods Phys. Res., Sect. A 376, 29 (1996).

[29] J. Altmann et al., in X-Ray Optics, Instruments, and Mission, edited by Richard B. Hoover and Arthur B. Walker, SPIE Proceedings Vol. 3444 (SPIE-International Society for Optical Engineering, Bellingham, WA, 1998), p. 350; J. W. Egle et al., ibid., p. 359; P. Friedrich et al., ibid., p. 369.

[30] H. Schlattl, A. Weiss, and G. Raffelt, Astropart. Phys. 10, 353 (1999).

[31] D. B. Kaplan, Nucl. Phys. B260, 215 (1985). 